\title{
The gut microbiome and its interaction with health, disease, treatment response and toxicity in patients advanced cancer: focus on lung cancer and immunotherapy
}

\author{
Nadina Tinsley ${ }^{1}$, Natalie Cook $^{1,2}$ \\ ${ }^{1}$ The Christie NHS Foundation Trust, Manchester, UK; ${ }^{2}$ Division of Cancer Sciences, University of Manchester, Manchester, UK \\ Correspondence to: Dr. Natalie Cook. Honorary Consultant in Medical Oncology, Experimental Cancer Medicine Team (ECMT), The Christie NHS \\ Foundation Trust, Wilmslow Road, Manchester M20 4BX, UK. Email: Natalie.Cook@Christie.nhs.uk. \\ Comment on: Katayama Y, Yamada T, Shimamoto T, et al. The role of the gut microbiome on the efficacy of immune checkpoint inhibitors in \\ Japanese responder patients with advanced non-small cell lung cancer. Transl Lung Cancer Res 2019;8:847-53.
}

Submitted Mar 09, 2020. Accepted for publication May 19, 2020.

doi: $10.21037 /$ tlcr-20-407

View this article at: http://dx.doi.org/10.21037/tlcr-20-407

The microbiome comprises a complex community of microorganisms that live symbiotically within hosts. In humans, the gut microbiome has the largest numbers of bacteria and the greatest number of species compared to other areas of the body (1). Research within this area has shown that both have co-evolved in a mutualistic relationship known to generate health and disease (2).

Changes in the microbiome flora have been shown to be implicated in the development of a diverse range of diseases such as obesity, type 2 diabetes, cardiovascular disease and autoimmunity such as inflammatory bowel disease, asthma, rheumatoid arthritis (2).

In the field of Oncology, specific bacteria have been shown to be involved in carcinogenesis. The microbiome has also been shown to influence the efficacy and toxicity of some anti-cancer therapy (2). Preclinical and early clinical data suggest that modifying the host's microbiome could improve the efficacy of immunotherapy for cancer and become a novel biomarker for modulating and enhancing response, especially in patients treated with CTLA-4 and PD-(L)1 checkpoint blockade (3-7).

Metagenomic studies to date have primarily used 2 ways of identifying particular bacterial communities. The most common low cost and high throughput method is selective amplification and sequencing of a part of the gene encoding part of the small ribosomal subunit in this species, the $16 \mathrm{~S}$ ribosomal RNA. This method is usually limited to a family or genus level. The second most commonly used method is metagenomic shotgun sequencing which generates short reads representing the whole genomic content. This is considered superior to $16 \mathrm{~S}$ rRNA sequencing as it can identify down to species level and can also be used to characterise non-bacterial organisms (2).

A number of studies to date have shown a correlation between the gut microbiome and demonstrated that this can influence the effects of immunotherapy and some chemotherapy. An early mouse study showed the gut microbiome is essential for optimal responses to CpGoligonucleotide immunotherapy which activates immune cells through toll like receptor 9 signaling (8). Another mouse model showed that Cyclophosphomide alters the composition of microbiome in the small intestine and induces the translocation of species of $\mathrm{Gram}^{+}$bacteria into secondary lymphoid organs. These bacteria stimulate the generation of a specific subset of "pathogenic" T helper 17 (pTh17) cells and memory Th1 immune responses which influence the response to cyclophosphomide (9).

Pioneering work in the field of immuno-oncology with CTLA-4 and PD-(L)1 checkpoint blockade found that the anti-tumour effects of these agents are modulated by distinct bacterial species. Tumours in antibiotic treated or germ free mice did not respond to checkpoint blockade and this primary resistance was overcome by gavage of specific micro-organisms or faecal microbial transplant. These studies established the link between the microbiome and immune checkpoint blockade and inspired clinical quests to add to the growing evidence, in particular further correlative metagenomic analyses (3-7). 
Katayama et al. investigated the role of the gut microbiome on the efficacy of immune checkpoint inhibitors (ICIs) in a small single center cohort of 17 Japanese patients (10). This study included patients diagnosed with non-small cell lung cancer (NSCLC) and only included patients who had received treatment with ICIs for over 3 months. The authors collected stool samples on one occasion between June 2017 and March 2018 from each patient and performed $16 \mathrm{~S}$ rRNA, performing statistical analyses and correlating these with response defined as partial response to treatment according to RECIST 1.1 or no response [stable or progressive disease (PD)]. Results from the study revealed the gut microbiomes of responders were significantly rich with Lactobacillus, Clostridium, and Syntrophococcus when compared to non-responders. Gut microbiomes of the non-responders were significantly rich with Bilopbila, Sutterella, and Parabacteroides. Patients with a high abundance of Lactobacillus, Clostridium, and Syntrophococcus tended to have a longer time to treatment failure (TTF). Patients with a low amount of Bilophila and Sutterella had a significantly prolonged TTF. Unlike other published studies, the alpha-diversity of the gut microbiota was not significantly different between the responders and non-responders and did not influence the TTF. However, the absolute numbers to achieve statistical significance are very low.

Previously published studies have revealed a specific bacterial landscape that appears more common in patients who respond to treatment, whereas other bacterial sequences appear to be over-represented in non-responder patients. These discrepancies could be multifactorial, ranging from inter-patient variability due to previous differing therapy, medications, diet, geographic location or other genetic factors. The recurrent species which appear to be correlated with response across studies are Faecalibacterium, Bacteroidales, Ruminococcaceae, Clostridiales. Not all of these are mentioned in this study (11).

Katayama and colleagues did not give insights on sequential testing of samples which can change throughout time and could potentially generate a differing response to immunotherapy or lead to treatment failure. It would be interesting to know whether the microbiome is any different at treatment failure compared to at the start and what could lead for this dysbiosis to happen.

It could also be seen as biased towards responders in view of the inclusion of patients on treatment for over 3 months. In addition, the group selected had generally a good performance status, PS $0-1$ which could also select bias towards patients who will overall be more likely to benefit. This can be seen in the higher response rates achieved compared to responses seen in other studies. The authors very astutely took into account use of corticosteroids and antibiotics in their analysis however the numbers are too small to look for any significant differences. There is no mention of whether patients with brain metastases were included in this study.

Stable or progressive disease (as per RECIST criteria) at the time of $1^{\text {st }}$ clinical evaluation on ICIs could be difficult to interpret in cases of pseudo-progression. The use of irRECIST is not mentioned. A number of patients with stable disease were classed as non-responders however if these patients had prolonged periods of stable disease it is possible they could be responding patients.

Given the complexity of the gut microbiome and its interaction with health, disease, treatment response and toxicity, it can be complex to translate definite results into patient outcomes. Even if individuals possess the same bacterial strain, there can be differences in how these interact with the rest of the microbiome, therefore caution is advised when assigning attributes to particular bacteria. A recent trial which led to $E$. Coli septicaemia post Faecal microbial transplant has recently re-started after a Suspected Unexpected Serious Adverse Reaction (SUSAR) highlighting the importance of remaining vigilant when researching new therapies (12). In addition, studies have focused on the bacterial components of the microbiome and not on fungi, viruses or protozoa.

Sadly, despite attempts to understand and enhance immunotherapy responses, cancer cells grow and mutate with different therapies and it likely that the microbiome is just one of the factors at interplay. Further large scale prospective studies investigating the gut microbiome and its effect efficacy of ICIs are ongoing and results are eagerly awaited.

\section{Acknowledgments}

Funding: None.

\section{Footnote}

Provenance and Peer Review: This article was commissioned by the editorial office, Translational Lung Cancer Research. The article did not undergo external peer review.

Conflicts of Interest: Both authors have completed the 
ICMJE uniform disclosure form (available at http://dx.doi. org/10.21037/tlcr-20-407). NC reports other from Roche, other from Astra zeneca, personal fees and other from Tarveda, personal fees and other from RedX, other from Taiho, other from Eisai, other from Boeringer, outside the submitted work. NT has no conflicts of interest to declare.

Ethical Statement: The authors are accountable for all aspects of the work in ensuring that questions related to the accuracy or integrity of any part of the work are appropriately investigated and resolved.

Open Access Statement: This is an Open Access article distributed in accordance with the Creative Commons Attribution-NonCommercial-NoDerivs 4.0 International License (CC BY-NC-ND 4.0), which permits the noncommercial replication and distribution of the article with the strict proviso that no changes or edits are made and the original work is properly cited (including links to both the formal publication through the relevant DOI and the license). See: https://creativecommons.org/licenses/by-nc-nd/4.0/.

\section{References}

1. Quigley EMM. Gut bacteria in health and disease. Gastroenterol Hepatol (N Y) 2013;9:560-9.

2. Fessler J, Matson V, Gajewski TF. Exploring the emerging role of the microbiome in cancer immunotherapy. $\mathrm{J}$ Immunother Cancer 2019;7:108.

3. Vétizou M, Pitt JM, Daillère R, et al. Anticancer immunotherapy by CTLA-4 blockade relies on the gut microbiota. Science 2015;350:1079-84.

Cite this article as: Tinsley N, Cook N. The gut microbiome and its interaction with health, disease, treatment response and toxicity in patients advanced cancer: focus on lung cancer and immunotherapy. Transl Lung Cancer Res 2020;9(6):2305-2307. doi: $10.21037 /$ tlcr-20-407
4. Routy B, Le Chatelier E, Derosa L, et al. Gut microbiome influences efficacy of PD-1-based immunotherapy against epithelial tumors. Science 2018;359:91-7.

5. Sivan A, Corrales L, Hubert N, et al. Commensal Bifidobacterium promotes antitumor immunity and facilitates anti-PD-L1 efficacy. Science 2015;350:1084-9.

6. Gopalakrishnan V, Spencer CN, Nezi L, et al. Gut microbiome modulates response to anti-PD-1 immunotherapy in melanoma patients. Science 2018;359:97-103.

7. Matson V, Fessler J, Bao R, et al. The commensal microbiome is associated with anti-PD-1 efficacy in metastatic melanoma patients. Science 2018;359:104-8.

8. Iida N, Dzutsev A, Stewart CA, et al. Commensal bacteria control cancer response to therapy by modulating the tumor microenvironment. Science 2013;342:967-70.

9. Viaud S, Saccheri F, Mignot G, et al. The intestinal microbiota modulates the anticancer immune effects of cyclophosphamide. Science 2013;342:971-6.

10. Katayama Y, Yamada T, Shimamoto T, et al. The role of the gut microbiome on the efficacy of immune checkpoint inhibitors in Japanese responder patients with advanced non-small cell lung cancer. Transl Lung Cancer Res 2019;8:847-53.

11. Gong J, Chehrazi-Raffle A, Placencio-Hickok V, et al. The gut microbiome and response to immune checkpoint inhibitors: preclinical and clinical strategies. Clin Transl Med 2019;8:9.

12. DeFilipp Z, Bloom PP, Torres Soto M, et al. DrugResistant E. coli Bacteremia Transmitted by Fecal Microbiota Transplant. N Engl J Med 2019;381:2043-50. 